\section{Preventing Nuclear Artifact in Hematologic Specimens}

Robert Santoianni

Emory University Hospital, Atlanta, Georgia

\section{Abstract}

The bubbled, popcorn or moth-eaten appearance of nuclei hampered accurate microscopic evaluation of bone marrow and lymph node biopsies. This artifact was also noticed in skin biopsies, but had little bearing on diagnosis. All aspects of specimen handling processing and staining were scrutinized and improved morphological detail was attained only after blocks were recut and allowed to dry completely before staining.

\section{Introduction}

Turnaround time is an important facet of our quality improvement plan. We constantly peruse ways to expedite results without compromising quality. A common attempt to "cut corners" by some histology laboratories is to shorten the drying time of slides between sectioning and staining. To facilitate this, we increased the drying temperature from 60 degrees $C$ to 70-80 degrees $C$ and noticed the appearance of a nuclear artifact, primarily in bone marrow and lymph node specimens, which hampered diagnosis. Recuts of the same blocks had no nuclear artifact. We examined the methodology of our workflow and realized recuts were dried differently than initial sections and the problem was not the increased temperature, but the length of time it took to completely remove the water layer between the section and slide.

\section{Materials and Methods}

Bone marrow cores were fixed in B5 for 3-4 hours, washed in tap water and demineralized in RDO (APEX Engineering Products Corp., Plain-

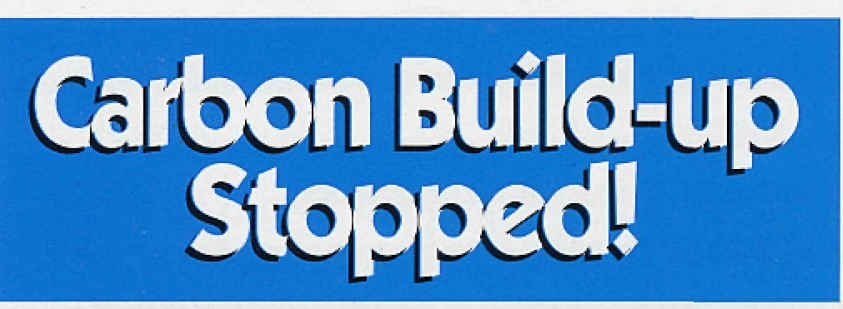

SEM Laboratory Secret Revealed:
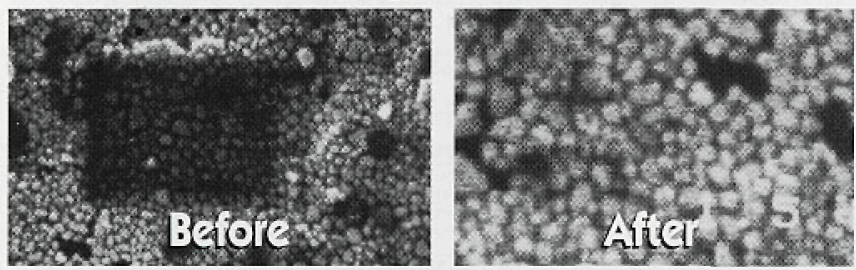

SEM manufacturers won't admit it, but most SEMs are subject to contamination build-up - even dry pumped systems. To stop hydrocarbon condensation, smart SEM users rely on the XEI Scientific SEM-CLEAN ${ }^{\mathrm{Tx}}$ system.

Result: Outstanding pictures at low $\mathrm{kV}$ and high resolution and no oil on EDS X-ray detector windows. The Nitrogen purge of the inexpensive SEM-CLEAN system cleans your electron microscope while you're away.

\section{SEM-CLEAN"' Stops the Oil}

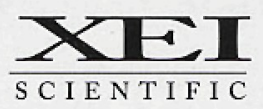

3124 Wessex Way, Redwood City, CA 94061-1348 650-369-0133 - Fax 650-363-1659

http://www.msa.microscopy.com/SM/XEI/XEIHomePage.html field, IL). The fixed, demineralized specimens were held in running tap water until they could be included in the overnight processing run along with $\mathrm{B} 5$ and formalin fixed lymph nodes. The run included additional fixation in neutral buffered formalin, dehydration through graded alcohol, clearing in xylene and infiltration in Paraplast $X$-tra (Monoject Scientific, St. Louis, MO). Specimens were embedded in Paraplast X-tra, cooled, and sectioned at $3 \mu \mathrm{m}$ on either a Leitz 1512 or AO 820 microtome. Slides were dried for 30 minutes at 60 degrees.C and stained in the usual manner. Our modification was to dry freshly cut sections for one hour at 37 degrees $C$ and then transfer to 60 degrees.C for 40 minutes before staining'.

\section{Results}

Complete drying of sections consistently eliminated the bubble/popcorn artifact.

\section{Discussion}

Since the publication of the original article in 1990, we have made improvements to our processing and staining protocols. Initial fixation and demineralization remain the same. The processing run now includes additional fixation in Penfix (Richard-Allan Scientific Kalamazoo, MI) clearing in Clear Rite 3 (Richard-Allan Scientific, Kalamazoo, MI), infiltration in Infiltration Medium (Surgipath Medical Industries, Inc., Richmond, LL) and embedding in EM-40O Embedding Medium (Surgipath Medical Industries, Inc., Richmond, IL). Drying remains the same except that the acquisition of a new stainer (Jung Autostainer $\mathrm{XL}$, Leica, Inc., Deerfield, IL) which has a built-in dryer lengthens the drying time to 10 minutes.

This subtle artifact remains overlooked by surgical pathologists, who are less concerned with nuclear detail than hematopathologists. In the past it went unnoticed or acknowledged as a preprocessing handling problem.

Berard suggests that water is absorbed from the tissue float bath by nuclei in freshly cut sections ${ }^{2}$. If the water is not evaporated completely by air drying or slide dryer, distortion of nuclear detail will result. This explanation helped us solve the puzzle of artifact-free recuts: these recuts were performed by histotechs later in the day, and slides were left to air dry longer than in the busy morning.

The adjustment to the hematologic specimen protocol added slightly more time to the process, but consistently artifact-free sections are well worth the effort, ultimately improving the efficiency of the laboratory as well as the quality of the work.

The sporadic appearance and limited importance of the artifact in surgical pathology does not warrant a change in protocol for routine specimens.

\section{References}

1 Santoianni, $H_{\text {., }}$ and Hammami, A.: Nuclear Bubbling: An Overlooked Artifact. The Journal of Histotechnology Vol.13, No.2, June 1990.

2. Berard, C., Bowling, M.; Technical Factors in Evaluation of Lymph Node Biopsies. Tutorial on Neoplastic Hematopathology, Universal City, CA, February 1989.

3. Beard, C., Nabers, K., Bowling, M., Berard, C.: Achieving Technical Excellence in Lymph Node Specimens: An Update. Laboratory Medicine 16:468475,1985 .

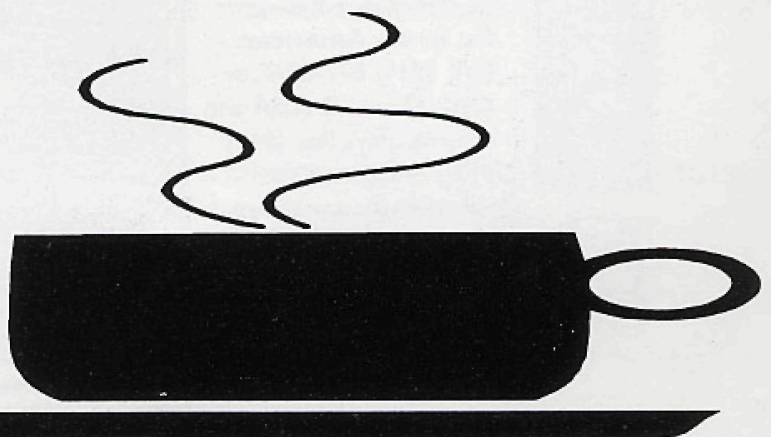

